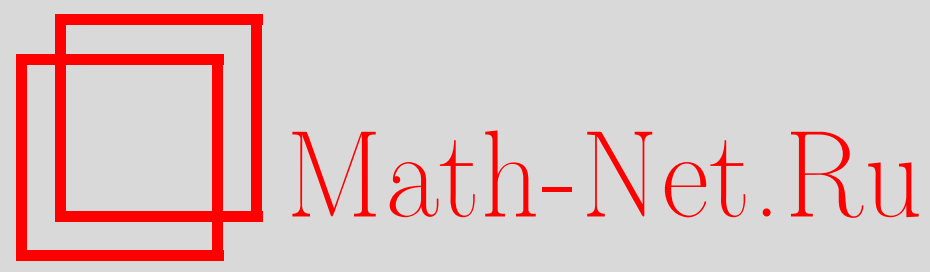

Р. С. Юлмухаметов, В. В. Напалков, О преобразовании Гильберта в пространстве Бергмана, Матем. заметки, 2001, том 70, выпуск 1, 68-78

DOI: https://doi.org/10.4213/mzm720

Использование Общероссийского математического портала Math-Net.Ru подразумевает, что вы прочитали и согласны с пользовательским соглашением http://www.mathnet.ru/rus/agreement

Параметры загрузки:

IP : 52.90 .164 .192

26 апреля 2023 г., $17: 24: 55$

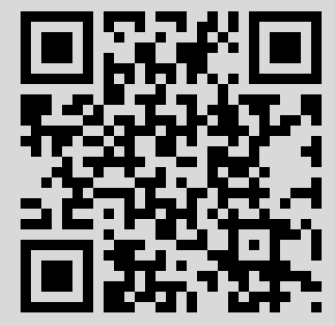




\section{О ПРЕОБРАЗОВАНИИ ГИЛЬБЕРТА \\ В ПРОСТРАНСТВЕ БЕРГМАНА}

\section{В.В. Напалков (мл.), Р. С. Юлмухаметов}

В работе описан образ оператора преобразования Гильберта для пространства Бергмана.

Библиографоия: 16 названий.

Пусть $X$ - функциональное топологическое пространство аналитических функций в области $G \subset \overline{\mathbb{C}}$. Термин “функциональное” означает, что функционал $\delta_{z}: f \rightarrow f(z)$, заданный на $X$, является линейным и непрерывным для любого $z \in G$. Через $X^{*}$ обозначим совокупность линейных и непрерывных функционалов над $X$. Во многих задачах возникает необходимость иметь более полную информацию о $X^{*}$. Эту информацию можно получить, изучая различные представления $X^{*}$. Для представления $X^{*}$ можно использовать любую полную систему $\mathscr{F}=\left\{f_{\alpha}\right\}_{\alpha \in A}$, где $A$ - множество индексов. Обозначим через $L_{\mathscr{F}}$ следующий оператор: каждому $S \in X^{*}$ ставится в соответствие функция

$$
S\left(f_{\alpha}\right): A \rightarrow \mathbb{C}
$$

Из полноты системы $\mathscr{F}$ и теоремы Банаха следует инъективность отображения $L_{\mathscr{F}}:$ $X^{*} \rightarrow \mathbb{C}^{A}$, где $\mathbb{C}^{A}$ - множество комплекснозначных функций от переменного $\alpha \in A$. Для эффективного использования такого представления линейных и непрерьвных функционалов на $X$ необходимо решить две задачи:

1) определить образ $\operatorname{Im} L_{\mathscr{F}}$;

2) описать топологию в образе, наведенную сильной топологией сопряженного к $X$. В качестве примеров можно рассмотреть следующие.

1) В качестве $\mathscr{F}$ возьмем $\left\{(z-\xi)^{-} 1, \xi \in \mathbb{C} \backslash \bar{G}\right\}$. Как правило, полнота этой системы имеет место. В этом случае $L_{\mathscr{F}}-$ преобразование Коши. Очень просто выясняется, что $\operatorname{Im} L_{\mathscr{F}}$ лежит в $H_{0}(\overline{\mathbb{C}} \backslash \bar{G})$. Более детальные результаты получены в [1]-[5].

2) $G$ - вьпуклая ограниченная область, система $\mathscr{F}=\{\exp (\lambda z), \lambda \in \mathbb{C}\}$ полна в $X$. $L_{\mathscr{F}}$ в этом случае назьвается преобразованием Фурье-Лапласа функиионала из $X^{*}$. В этом случае $\operatorname{Im} L_{\mathscr{F}} \subset H(\mathbb{C})$. Для пространства $X=H(G)$ эта задача исследовалась Полиа (см., например, [6]). Для пространств аналитических в $G$ функций, имеющих определенньй рост вблизи границы, см. работы [7]-[9].

Первый автор поддержан грантом "Ведушие научные школы России" № 96-15-96196. Второй автор поддержан грантом Российского фонда фундаментальных исследований № 99-01-00159.

(C) В.В. НАПАлков (мл.), Р. С. Юлмухаметов 
3) $G=\{z \in \mathbb{C}:|z|<1\}, \mathscr{F}=\left\{z^{n}, n \in \mathbb{N}\right\}$. В этом случае $\operatorname{Im} L_{\mathscr{F}}$ пространство последовательностей.

Здесь мы рассмотрим пространство Бергмана, состоящее из функций, голоморфных в односвязной области $G \in \overline{\mathbb{C}}$ с жордановой границей и суммируемых в этой области с квадратом модуля по площади:

$$
B_{2}(G)=\left\{f \in H(G):\|f\|_{B_{2}(G)}^{2}=\int_{G}|f(z)|^{2} d v(z)<\infty\right\} .
$$

Это гильбертово пространство со скалярньм произведением

$$
(f, g)=\int_{G} f(z) \overline{g(z)} d v(z) .
$$

В качестве системы $\mathscr{F}$ возьмем систему $\left\{(z-\xi)^{-2}, \xi \in \mathbb{C} \backslash \bar{G}\right\}$. Задача об описании $B_{2}^{*}(G)$ в терминах преобразования Гильберта близка к задаче об описании $B_{2}^{*}(G)$ в терминах преобразования Коши, однако первая постановка допускает неограниченные области $G$.

Ранее вопрос об описании $B_{2}^{*}(G)$ в терминах преобразования Коши изучался в [4], где было получено описание $B_{2}^{*}(G)$ для ограниченных областей $G$ с границей класса $C^{1+0}$. В работе [10] этот результат был распространен на случай, когда $G$ - ограниченный квазикруг.

В данной работемы получим критерий возможности описания $B_{2}^{*}(G)$ в терминах преобразования Гильберта. Заметим, что близкие к теме данной статьи вопросы рассмотрены в [11], [12], где изучались свойства интеграла Коши на липшицевых кривых.

Лемма 1. Система функиий от переменной $z\left\{(z-\xi)^{-2}, \xi \in \mathbb{C} \backslash \bar{G}\right\}$ полна в пространстве $B_{2}(G)$.

ДокАЗАтЕЛЬСтво. Принадлежность системы $\left\{(z-\xi)^{-2}, \xi \in \mathbb{C} \backslash \bar{G}\right\}$ пространству $B_{2}(G)$ достаточно очевидна. Докажем полноту этой системы. Рассмотрим два случая:

1) $G$ - ограниченная односвязная область в $\overline{\mathbb{C}}$;

2) $G$ - неограниченная односвязная область в $\overline{\mathbb{C}}$.

1) Используем теорему Банаха. Из равенства

$$
\int_{G} \frac{\overline{g(z)}}{(z-\xi)^{2}} d v(z)=0, \quad \xi \in \mathbb{C} \backslash \bar{G},
$$

следует

$$
\int_{G} \frac{\overline{g(z)}}{z-\xi} d v(z)=C, \quad \xi \in \mathbb{C} \backslash \bar{G},
$$

где $C$ - постоянная. Левая часть последнего равенства стремится к 0 при $\xi \rightarrow \infty$, поэтому $C=0$. Так как система $\left\{(z-\xi)^{-1}, \xi \in \mathbb{C} \backslash \bar{G}\right\}$ полна в $B_{2}(G)[13]$, то $g(z) \equiv 0$. По теореме Банаха система $\left\{(z-\xi)^{-2}, \xi \in \mathbb{C} \backslash \bar{G}\right\}$ полна в $B_{2}(G)$.

2) Этот случай сводится к первому конформной заменой переменных $w=1 / z, \eta=1 / \xi$.

Каждому линейному непрерьвному функционалу $S$ на $B_{2}(G)$ поставим в соответствие функцию

$$
\widetilde{S}(\xi)=S\left(\frac{1}{(z-\xi)^{2}}\right), \quad \xi \in \mathbb{C} \backslash \bar{G},
$$

которая называется преобразованием Гильберта функиионала $S$.

Справедлива следующая теорема. 
ТЕорема 1. Пусть $G \in \overline{\mathbb{C}}$ - односвязная область с жсордановой граниией, $S$ линейный непрерывный функционал на $B_{2}(G)$ и

$$
\gamma(\xi)=\frac{1}{\pi} S_{z}\left(\frac{1}{(z-\xi)^{2}}\right), \quad \xi \in \mathbb{C} \backslash \bar{G} .
$$

Тогда

$$
\|\gamma\|_{B_{2}(\mathbb{C} \backslash \bar{G})} \leqslant\|S\|_{B_{2}^{*}(G)}
$$

ДокАЗАТЕЛЬСтво. Пусть функционал $S$ порождается функцией $g \in B_{2}(G)$

$$
S(f)=\int_{G} f(z) \overline{g(z)} d v(z), \quad\|S\|_{B_{2}^{*}(G)}=\|g\|_{B_{2}(G)} .
$$

Тогда

$$
\gamma(\xi)=\frac{1}{\pi} \int_{G} \frac{\overline{g(z)}}{(z-\xi)^{2}} d v(\xi), \quad \xi \in \mathbb{C} \backslash \bar{G} .
$$

Рассмотрим сингулярный интегральный оператор $\mathbb{T}$ на $L_{2}(\mathbb{C})$

$$
\mathbb{T} u(\xi)=\frac{1}{\pi} \lim _{\varepsilon \rightarrow 0} \int_{|z-\xi| \geqslant \varepsilon} \frac{u(z)}{(z-\xi)^{2}} d v(z) .
$$

Предельньй переход понимается в смысле $L_{2}(\mathbb{C})$. Вводя функцию $\Omega(w)=|w|^{2} /\left(\pi w^{2}\right)$, этот оператор запишем в виде

$$
\mathbb{T} u(\xi)=\lim _{\varepsilon \rightarrow 0} \int_{|z-\xi| \geqslant \varepsilon} \frac{u(z)}{|z-\xi|^{2}} \Omega(z-\xi) d v(z) .
$$

$\Phi$ ункцию $\Omega$ от одного комплексного переменного можно понимать как функцию от двух вешественньх переменных. Эта функция удовлетворяет условиям следующей теоремы из работы [13, с. 52].

Теорема А. Пусть функиия $\Omega: \mathbb{R}^{2} \rightarrow \mathbb{C}$ однородна степени 0 и удовлетворяет условию Липиица

$$
\left|\Omega\left(x_{1}\right)-\Omega\left(x_{2}\right)\right| \leqslant M\left|x_{1}-x_{2}\right|^{\alpha}, \quad\left|x_{1}\right|=\left|x_{2}\right|=1, \quad \exists \alpha>0,
$$

$u$

$$
\int_{|x|=1} \Omega(x) d \sigma(x)=0
$$

әде $d \sigma(x)$ - әлемент длины дуги окружности. Для $f \in L_{2}\left(\mathbb{R}^{2}\right)$ положим

$$
\mathbb{T}_{\varepsilon}(f)(x)=\int_{|x-y| \geqslant \varepsilon} \frac{\Omega(x-y)}{|x-y|^{2}} f(y) d y .
$$

Tогда

1) сущ,ствует константа $A$ (не зависящая от $f$ и $е$ ) такая, что

$$
\left\|\mathbb{T}_{\varepsilon}(f)\right\|_{L_{2}\left(\mathbb{R}^{2}\right)} \leqslant A\|f\|_{L_{2}\left(\mathbb{R}^{2}\right)}
$$


2) предел $\lim _{\varepsilon \rightarrow 0} \mathbb{T}_{\varepsilon}(f)=\mathbb{T}(f)$ существует в смислле $L_{2}\left(\mathbb{R}^{2}\right)$ и

$$
\|\mathbb{T}(f)\|_{L_{2}\left(\mathbb{R}^{2}\right)} \leqslant A\|f\|_{L_{2}\left(\mathbb{R}^{2}\right)}
$$

3) преобразования Фурье от $f u \mathbb{T}(f)$ связаны соотношением

$$
(\widehat{\mathbb{T} f})(x)=m(x) \hat{f}(x),
$$

где $m$ - однородная функиия степени 0 и

$$
m(x)=\int_{|y|=1}\left(\frac{\pi i}{2} \operatorname{sign}(x \cdot y)+\ln \frac{1}{|x \cdot y|}\right) \Omega(y) d \sigma(y), \quad|x|=1 .
$$

Применяя эту теорему, получим, что $\mathbb{T}$ непрерьвно действует на $L_{2}\left(\mathbb{R}^{2}\right)$ и для преобразований $Ф$ урье верно соотношение

$$
(\widehat{\mathbb{T} u})(\xi)=m(\xi) \hat{u}(\xi), \quad \xi \in \mathbb{C},
$$

где

$$
m(\xi)=\int_{|z|=1}\left(\frac{\pi i}{2} \operatorname{sign}(\operatorname{Re}(z \bar{\xi}))+\ln \left(\frac{1}{|\operatorname{Re}(z \bar{\xi})|}\right)\right) \Omega(z) d \sigma(z),
$$

$d \sigma-$ элемент длины дуги. Прямьм подсчетом убеждаемся, что

$$
m(\xi)=e^{-2 i \arg (\xi)} .
$$

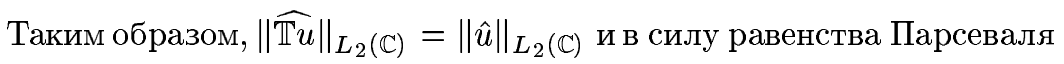

$$
\|\mathbb{T} u\|_{L_{2}(\mathbb{C})}=\|u\|_{L_{2}(\mathbb{C})},
$$

т.е. оператор $\mathbb{T}$ является изометрией $L_{2}\left(\mathbb{R}^{2}\right)$. Из соотношения $(1)$ видим, что $\gamma(\xi)$ можно понимать как сужение на $\mathbb{C} \backslash \bar{G}$ образа при отображении $\mathbb{T}$ функции $\bar{g}(z)$, продолженной нулем на всю плоскость, т.е.

$$
\gamma(\xi)=\mathbb{T} \bar{g}(\xi), \quad \xi \in \mathbb{C} \backslash \bar{G} .
$$

Тем самым, имеем

$$
\|\gamma\|_{B_{2}(\mathbb{C} \bar{G})}=\|\gamma\|_{L_{2}(\mathbb{C} \bar{G})} \leqslant\|g\|_{B_{2}(G)}=\|S\|_{B_{2}^{*}(G)}
$$

и теорема 1 доказана.

Таким образом, оператор преобразования Гильберта непрерывно действует из пространства $B_{2}^{*}(G)$ в пространство $B_{2}(\mathbb{C} \backslash \bar{G})$ и инъективен. Возникает вопрос: когда этот оператор сюръективен? Целью этой работы является доказательство следующего факта. 
ОПРЕДЕЛЕниЕ. Односвязная область $G \subset \overline{\mathbb{C}}$ с жордановой границей назьвается

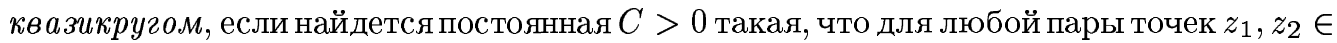
$\partial G$ выполнено соотношение

$$
\operatorname{diam} l\left(z_{1}, z_{2}\right) \leqslant C\left|z_{1}-z_{2}\right|
$$

где $l\left(z_{1}, z_{2}\right)$ - та из двух дуг множества $\partial G \backslash\left\{z_{1}, z_{2}\right\}$, которая имеет наименьший диаметр.

Теорема 2. Пусть $G$ - односвязная жсорданова область в $\overline{\mathbb{C}}$. Для того чтобъ оператор преобразования Гильберта, действующий из пространства $B_{2}^{*}(G)$ в $B_{2}(\mathbb{C} \backslash \bar{G})$, был сюрвективен, необходимо и достаточно, чтобы область $G$ была квазикругом.

Предварительно получим некоторые вспомогательные результаты.

Лемма 2. Пусть $g \in B_{2}(G)$. Продолжим ее нулем на всю комплексную плоскость. Тогда $\mathbb{T} \bar{g}(\xi) \in B_{2}(G)$.

ДокАЗАТЕЛьСтво. Действительно, то, что $\mathbb{T} \bar{g}(\xi) \in L_{2}(G)$, следует из теоремы А. Остается показать, что $\mathbb{T} \bar{g}(\xi)$ аналитична в $G$. Если $K$ произвольньй круг, содержащийся в $G$, и $\xi \in K$, то (см. [4])

$$
\int_{K} \frac{\overline{g(z)}}{(z-\xi)^{2}} d v(z)=0, \quad \xi \in K
$$

Поэтому

$$
\mathbb{T} \bar{g}(\xi)=\frac{1}{\pi} \int_{G} \frac{\overline{g(z)}}{(z-\xi)^{2}} d v(\xi)=\frac{1}{\pi} \int_{G \backslash K} \frac{\overline{g(z)}}{(z-\xi)^{2}} d v(\xi), \quad \xi \in K
$$

Из последнего равенства легко видеть, что функция $\mathbb{T} \bar{g}(\xi)$ аналитична в $K$. Поскольку круг $K$ произвольный, лежаший в $G$, то $\mathbb{T} \bar{g}(\xi)$ аналитична в $G$.

Для области $G$ определим операторы $\mathbb{T}_{1, G}$ и $\mathbb{T}_{2, G}$, которые действуют из пространства $\overline{B_{2}(G)}$ (здесь $\overline{B_{2}(G)}=\left\{f: \bar{f} \in B_{2}(G)\right\}$, а функцию $f$ считаем продолженной нулем на $\mathbb{C} \backslash \bar{G})$ :

$$
\begin{aligned}
& \left(\mathbb{T}_{2, G} f\right)(\xi) \stackrel{\text { def }}{=} \begin{cases}0, & \xi \in G, \\
(\mathbb{T} f)(\xi), & \xi \in \mathbb{C} \backslash \bar{G},\end{cases} \\
& \left(\mathbb{T}_{1, G} f\right)(\xi) \stackrel{\text { def }}{=}(\mathbb{T} f)(\xi)-\left(\mathbb{T}_{2, G} f\right)(\xi) .
\end{aligned}
$$

Если $\gamma$ - преобразование Гильберта функционала из $B_{2}^{*}(G)$, порождаемого функцией $g \in B_{2}(G)$, то

$$
\gamma(\xi)=\left(\mathbb{T}_{2, G} \bar{g}\right)(\xi), \quad \xi \in \mathbb{C} \backslash \bar{G} .
$$

Для произвольной односвязной области $G$ оператор $\mathbb{T}_{2, G}$ ограничен как оператор из $\overline{B_{2}(G)}$ в $B_{2}(\mathbb{C} \backslash \bar{G})$, а оператор $\mathbb{T}_{1, G}$ действует из $\overline{B_{2}(G)}$ в $B_{2}(G)$, причем $\mathbb{T}_{1, D} \equiv 0$ $(D=\{z \in \mathbb{C}:|z|<1\})$ (см. [4]).

Сюръективность преобразования Гильберта равносильна сюръективности оператоpa $\mathbb{T}_{2, G}$. Справедлива следуюшая 
Лемма 3. Oператор $\mathbb{T}_{2, G}: \overline{B_{2}(G)} \rightarrow B_{2}(\mathbb{C} \backslash \bar{G})$ сюрдективен тогда и только тогда, когда $\left\|\mathbb{T}_{1, G}\right\|<1$.

ДокАЗАтЕльство. Необходимость. Если $\mathbb{T}_{2, G}$ сюръективен, то этот оператор взаимно однозначен и, следовательно, по теореме об открытом отображении существует непрерьвньй обратный оператор $\mathbb{T}_{2, G}^{-1}$. Если $C=\left\|\mathbb{T}_{2, G}^{-1}\right\|$, то для любого $g \in B_{2}(G)$ имеем

$$
\|\bar{g}\|_{\overline{B_{2}(G)}}=\left\|\mathbb{T}_{2, G}^{-1} \mathbb{T}_{2, G}(\bar{g})\right\|_{\overline{B_{2}(G)}} \leqslant C\left\|\mathbb{T}_{2, G}(\bar{g})\right\|_{B_{2}(\mathbb{C} \bar{G})}
$$

И

$$
\left\|\mathbb{T}_{2, G}(\bar{g})\right\|_{B_{2}(\mathbb{C} \bar{G})} \geqslant \frac{1}{C}\|\bar{g}\|_{\overline{B_{2}(G)}} .
$$

Так как

$$
\begin{aligned}
\|\bar{g}\|_{B_{2}(G)}^{2} & =\|\mathbb{T} \bar{g}\|_{L_{2}(\mathbb{C})}^{2}=\left\|\mathbb{T}_{1, G}(\bar{g})\right\|_{B_{2}(G)}^{2}+\left\|\mathbb{T}_{2, G}(\bar{g})\right\|_{B_{2}(\mathbb{C} \backslash \bar{G})}^{2} \\
& \geqslant\left\|\mathbb{T}_{1, G}(\bar{g})\right\|_{B_{2}(G)}^{2}+\frac{1}{C^{2}}\|\bar{g}\|_{B_{2}(G)}^{2}
\end{aligned}
$$

то

$$
\left\|\mathbb{T}_{1, G}(\bar{g})\right\|_{B_{2}(G)}^{2} \leqslant\left(1-\frac{1}{C^{2}}\right)\|\bar{g}\|_{B_{2}(G)}^{2}
$$

и, следовательно, $\left\|\mathbb{T}_{1, G}\right\| \leqslant\left(1-1 / C^{2}\right)^{1 / 2}<1$.

Достаточность. Пусть $\left\|\mathbb{T}_{1, G}\right\|<1$. Тогда из равенства

$$
\|\bar{g}\|_{B_{2}(G)}^{2}=\left\|\mathbb{T}_{1, G}(\bar{g})\right\|_{B_{2}(G)}^{2}+\left\|\mathbb{T}_{2, G}(\bar{g})\right\|_{B_{2}(\mathbb{C} \bar{G})}^{2}
$$

получаем, что найдется $C>0$ такая, что

$$
C\|\bar{g}\|_{B_{2}(G)} \leqslant\left\|\mathbb{T}_{2, G} \bar{g}\right\|_{B_{2}(\mathbb{C} \bar{G})} \quad \forall g \in B_{2}(G) .
$$

Образ $\operatorname{Im}\left(\mathbb{T}_{2, G}\right)$ плотен в $B_{2}(\mathbb{C} \backslash \bar{G})$. Действительно, функционал $\delta_{z}: f \rightarrow f(z), z \in G$, $f \in B_{2}(G)$, принадлежит $B_{2}^{*}(G)$. Следовательно, при фиксированном $z \in G$ функция $\delta_{z}\left((\xi-\eta)^{-2}\right)=(\xi-z)^{-2} \in B_{2}(\mathbb{C} \backslash \bar{G})$ (теорема 1$)$, а система $\left\{(\xi-z)^{-2}, z \in G\right\}$ полна в $B_{2}(\mathbb{C} \backslash \bar{G})$ (лемма 1$)$.

По теореме Банаха в силу неравенства (3) оператор $\mathbb{T}_{2, G}$ сюръективен. Лемма 3 доказана.

Начиная доказательство теоремы 2 , выделим два случая:

1) $\infty \notin G$;

2) $\infty \in G$.

Проведем доказательство для случая 1 .

Однолистное конформное отображение $\varphi: D \rightarrow G$ единичного круга на $G$ порождает изометрию $R_{\varphi}: B_{2}(G) \rightarrow B_{2}(D)$, действующую по правилу

$$
g(z) \stackrel{R_{\varphi}}{\longrightarrow} g(\varphi(\xi)) \varphi^{\prime}(\xi)
$$

и соответственно изометрию $\bar{R}_{\varphi}: \overline{B_{2}(G)} \rightarrow \overline{B_{2}(D)}$, действующую по правилу

$$
\overline{g(z)} \stackrel{\bar{R}_{\varphi}}{\longrightarrow} \overline{g(\varphi(\xi)) \varphi^{\prime}(\xi)}
$$


Оператор $\mathbb{T}_{1, G}: \overline{B_{2}(G)} \rightarrow B_{2}(G)$ порождает оператор $A_{G}=R_{\varphi} \circ \mathbb{T}_{1, G} \circ \bar{R}_{\varphi}^{-1}$, действующий из $\overline{B_{2}(D)}$ в $B_{2}(D)$, причем, $\left\|A_{G}\right\|=\left\|\mathbb{T}_{1, G}\right\|$,

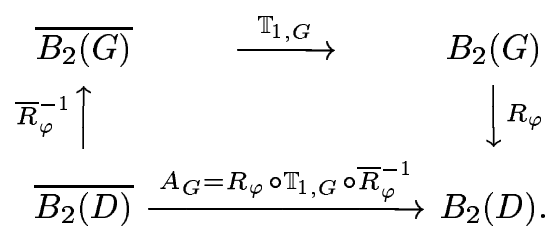

Нетрудно показать, что

$$
A_{G} \bar{g}(\xi)=\frac{1}{\pi} \int_{D} \overline{g(z)} \frac{\varphi^{\prime}(z) \varphi^{\prime}(\xi)}{(\varphi(z)-\varphi(\xi))^{2}} d v(z), \quad g \in B_{2}(D) .
$$

Поскольку $\mathbb{T}_{1, D} \equiv 0$ (см. [13]), то

$$
\begin{aligned}
A_{G} \bar{g}(\xi) & =A_{G} \bar{g}(\xi)-\mathbb{T}_{1, D} \bar{g}(\xi) \\
& =\frac{1}{\pi} \int_{D} \overline{g(z)}\left(\frac{\varphi^{\prime}(z) \varphi^{\prime}(\xi)}{(\varphi(z)-\varphi(\xi))^{2}}-\frac{1}{(z-\xi)^{2}}\right) d v(z) \\
& =\frac{1}{\pi} \int_{D} \overline{g(z)} \frac{\partial^{2}}{\partial \xi \partial z} \ln \frac{\varphi(z)-\varphi(\xi)}{z-\xi} d v(z) .
\end{aligned}
$$

Таким образом, лемму 3 можно сформулировать следующим образом.

Лемма $3^{\prime}$. Оператор преобразования Гильберта, действующий из пространства $B_{2}^{*}(G)$ в пространство $B_{2}(\mathbb{C} \backslash \bar{G})$, сюргективен тогда и только тогда, когда $\left\|A_{G}\right\|<1$.

Пусть $\varphi(z)=z+c_{1} z^{2}+\ldots$ - аналитическая в единичном круге $D$ функция. Необходимое и достаточное условие однолистности $\varphi$ в $D$ дает критерий Грунского (см., например, [14, с. 574]).

ТЕорема В (Грунский). Для того чтобы аналитическая в $D$ функиия вида $\varphi(z)=z+c_{1} z^{2}+\cdots$ была однолистна в $D$, необходимо и достаточно, чтобь выполнялось неравенство

$$
\left|\sum_{m, n=1}^{\infty} a_{m n} x_{n} x_{m}\right| \leqslant \sum_{n=1}^{\infty} \frac{\left|x_{n}\right|^{2}}{n}
$$

где числа $a_{m n}$ определяются из разложения функиии двух комплексных переменных $\ln ((\varphi(\xi)-\varphi(z)) /(\xi-z))$ в окрестности нуля в ряд Тейлора

$$
\ln \frac{\varphi(\xi)-\varphi(z)}{\xi-z}=\sum_{m, n=0}^{\infty} a_{m n} \xi^{m} z^{n}
$$

$a\left\{x_{n}\right\}_{n=0}^{\infty}-$ произвольная последовательность комплексных чисел, для которых правая часть неравенства сходится.

Класс однолистных и аналитических в $D$ функций $\varphi(z)=z+c_{1} z^{2}+\cdots$ обозначим через $S$. Через $S(k), k<1$, обозначим подмножество $S$, состоящее из функций, 
однолистных и аналитических в $D$, которые допускают продолжение до $k$ - квазиконформного автоморфизма расширенной комплексной плоскости $\overline{\mathbb{C}}$ (см., например, [15]), т.e.

1) в любом замкнутом прямоугольнике $\{x+i y: a \leqslant x \leqslant b ; c \leqslant y \leqslant d\} \subset \mathbb{C}$ функции $\operatorname{Re} \varphi(x+i y), \operatorname{Im} \varphi(x+i y)$ абсолютно непрерывны по $x \in[a, b]$ при почти всех $y \in[c, d]$ и абсолютно непрерьвны по $y \in[c, d]$ при почти всех $x \in[a, b]$;

2) $\partial \varphi / \partial z, \partial \varphi / \partial \bar{z} \in L_{2}^{\operatorname{loc}}(\mathbb{C})$;

3) функция $\varphi$ при почти всех $z \in \mathbb{C}$ удовлетворяет уравнению Бельтрами

$$
\frac{\partial \varphi}{\partial \bar{z}}=\mu(z) \frac{\partial \varphi}{\partial z}
$$

где $\mu(z)$ - комплекснозначная измеримая в $\mathbb{C}$ функция такая, что $\|\mu\|_{L_{\infty}(\mathbb{C})} \leqslant$ $k<1$.

ОПРЕДЕЛЕнИЕ. Будем говорить, что функция $\varphi(z)=z+c_{1} z^{2}+\cdots, z \in D$, удовлетворяет усиленному неравенству Грунского с константой $k<1$, если вьполнено условие

$$
\left|\sum_{m, n=1}^{\infty} a_{m n} x_{n} x_{m}\right| \leqslant k \sum_{n=1}^{\infty} \frac{\left|x_{n}\right|^{2}}{n}, \quad k<1,
$$

где $\left\{x_{n}\right\}_{n=0}^{\infty}-$ произвольная последовательность комплексных чисел, для которьх правая часть последнего неравенства сходится, а числа $a_{m n}$ определяются из (5).

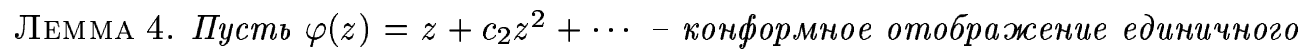
круга $D=\{z \in \mathbb{C}:|z|<1\}$ на область $G$. Oператор $A_{G}$ действует из пространства $\overline{B_{2}(D)}$ в $B_{2}(D)$,

$$
A_{G} f(\xi)=\frac{1}{\pi} \int_{D} f(z) \frac{\partial^{2}}{\partial \xi \partial z} \ln \frac{\varphi(z)-\varphi(\xi)}{z-\xi} d v(z) .
$$

Следуюшие условия әквивалентны:

1) функиия $\varphi$ удовлетворяет усиленному условию Грунского с константой $k<1$;

2) для любой функиии $f \in \overline{B_{2}(D)}$ выполнено условие

$$
\left|\left(A_{G} f, \bar{f}\right)_{B_{2}(D)}\right| \leqslant k\|f\|_{B_{2}(D)}^{2} .
$$

ДокАЗАТЕЛЬСТво. Любая последовательность комплексных чисел $\left\{x_{n}\right\}$, удовлетворяющая условию $\sum_{n=1}^{\infty}\left(\left|x_{n}\right|^{2} / n\right)<\infty$, порождает функцию из пространства $\overline{B_{2}(D)}$ :

$$
f(z) \stackrel{\text { def }}{=} \sum_{n=1}^{\infty} x_{n} \bar{z}^{n-1}, \quad z \in D, \quad\|f\|_{B_{2}(D)}^{2}=\pi \sum_{n=1}^{\infty} \frac{\left|x_{n}\right|^{2}}{n}<\infty .
$$

Обратно, всякая функция $f \in \overline{B_{2}(D)}$ может быть представлена в виде (6).

Непосредственным подсчетом убеждаемся, что неравенство

эквивалентно неравенству

$$
\left|\sum_{m, n=1}^{\infty} a_{m n} x_{n} x_{m}\right| \leqslant k \sum_{n=1}^{\infty} \frac{\left|x_{n}\right|^{2}}{n}, \quad k<1,
$$

$$
\left|\left(A_{G} f, \bar{f}\right)_{B_{2}(D)}\right| \leqslant k\|f\|_{B_{2}(D)}^{2}, \quad k<1,
$$

если $f(z)=\sum_{n=1}^{\infty} x_{n} \bar{z}^{n-1}, z \in D$. 
ДоКАЗАТЕЛЬСТво ТЕОРЕмЫ. Необходимость. Пусть $G$ - область, не являющаяся квазикругом, $\varphi$ - конформное отображение единичного круга $D$ на область $G$. Тогда найдется последовательность функций $f_{n}$ из пространства $\overline{B_{2}(D)},\left\|f_{n}\right\|=1$, такая, что

$$
\left|\left(A_{G} f_{n}, \bar{f}_{n}\right)_{B_{2}(D)}\right| \rightarrow 1 .
$$

Действительно, в противном случае $\varphi$ удовлетворяет усиленному условию Грунского с константой $k<1$. Тогда по теореме Поммеренке (см. [15]) найдется константа $k^{\prime}$, $k<k^{\prime}<1$, такая, что $\varphi \in S\left(k^{\prime}\right)$, и, следовательно, область $G$ - квазикруг [15]. Следовательно,

$$
\left\|A_{G}\right\| \geqslant \sup _{n}\left\|A_{G} f_{n}\right\| \geqslant \sup _{n}\left|\left(A_{G} f_{n}, \bar{f}_{n}\right)_{B_{2}(D)}\right| \geqslant 1 \text {. }
$$

Таким образом, по лемме $3^{\prime}$ оператор преобразования Гильберта не сюръективен.

Достаточность. Пусть область $G$ - квазикруг. Тогда конформное отображение единичного круга $D$ на область $G$ продолжается до $k$-квазиконформного автоморфизма расширенной комплексной плоскости $\overline{\mathbb{C}}($ см. [15], [16]). Следовательно, вьполняется усиленное неравенство Грунского с константой $k<1$ [15]. То есть справедливо соотношение

$$
\left|\left(A_{G} f, \bar{f}\right)_{B_{2}(D)}\right| \leqslant k\|f\|_{B_{2}(D)}^{2} \quad \forall f \in \overline{B_{2}(D)} .
$$

Рассмотрим оператор $\overline{A_{G}} f \stackrel{\text { def }}{=} \overline{A_{G} f}$. Этот оператор не является комплексно линейным. Однако он линеен над полем вещественных чисел. Кроме того, справедливо равенство $\left|\left(A_{G} f, \bar{f}\right)_{B_{2}(D)}\right|=\left|\left(\overline{A_{G}} f, f\right) \overline{B_{2}(D)}\right|$. Легко видеть, что $\left({\overline{A_{G}}}^{2} f, f\right) \frac{}{B_{2}(D)}=\left\|\overline{A_{G}} f\right\|_{B_{2}(D)}$. Нетрудно показать, что для любого $\lambda>0$

$$
\begin{aligned}
\left\|\overline{A_{G}} f\right\|_{B_{2}(D)}^{2}= & \frac{1}{4}\left(\left(\overline{A_{G}}\left(\lambda f+\frac{1}{\lambda} \overline{A_{G}} f\right), \lambda f+\frac{1}{\lambda} \overline{A_{G}} f\right)\right. \\
& \left.-\left(\overline{A_{G}}\left(\lambda f-\frac{1}{\lambda} \overline{A_{G}} f\right), \lambda f-\frac{1}{\lambda} \overline{A_{G}} f\right)\right) .
\end{aligned}
$$

Используя (7), оценим правую часть последнего равенства по модулю. Получим

$$
\begin{aligned}
\left\|\overline{A_{G}} f\right\|_{B_{2}(D)}^{2} & \leqslant \frac{k}{4}\left(\left\|\lambda f+\frac{1}{\lambda} \overline{A_{G}} f\right\|_{\overline{B_{2}(D)}}^{2}+\left\|\lambda f-\frac{1}{\lambda} \overline{A_{G}} f\right\|_{\overline{B_{2}(D)}}^{2}\right) \\
& =\frac{k}{2}\left(\lambda^{2}\|f\| \frac{2}{B_{2}(D)}+\frac{1}{\lambda^{2}}\left\|\overline{A_{G}} f\right\|_{B_{2}(D)}^{2}\right) .
\end{aligned}
$$

Правая часть последнего равенства достигает минимума при

$$
\lambda^{2}=\frac{\left\|\overline{A_{G}} f\right\|_{\overline{B_{2}(D)}}}{\|f\|_{\overline{B_{2}(D)}}} .
$$

Подставляя это значение в соотношение (9), получим

$$
\left\|\overline{A_{G}} f\right\|_{B_{2}(D)}^{2} \leqslant k\left\|\overline{A_{G}} f\right\|_{\overline{B_{2}(D)}}\|f\|_{\overline{B_{2}(D)}} .
$$


Следовательно, $\left\|A_{G}\right\| \leqslant k<1$. Поэтому в силу леммы $3^{\prime}$ оператор преобразования Гильберта, действующий из пространства $B_{2}^{*}(G)$ в пространство $B_{2}(\mathbb{C} \backslash \bar{G})$, сюръективен. Теорема 2 доказана для случая $\infty \notin G$.

Доказательство этой теоремы для случая $\infty \in G$ аналогично. Только нужно рассматривать конформные отображения внешности единичного круга $\mathbb{C} \backslash \bar{D}$ на область $G$. Функцию, осуществляющую это отображение, можно записать в виде

$$
\varphi(z)=z+a_{0}+\frac{a_{1}}{z}+\frac{a_{2}}{z^{2}}+\cdots
$$

Класс функций такого вида, мероморфных (с полюсом в $\infty$ ) и однолистных в $\mathbb{C} \backslash \bar{D}$, обозначим через $\Sigma$. Через $\Sigma(k), k<1$, обозначим подмножество $\Sigma$, состоящее из функций, однолистных и мероморфных в $\mathbb{C} \backslash \bar{D}$, которые допускают продолжение до $k$-квазиконформного автоморфизма $\overline{\mathbb{C}}$. Оператор $A_{G}$ в этом случае действует из $\overline{B_{2}(\mathbb{C} \backslash \bar{D})}$ в $B_{2}(G)$ и имеет вид

$$
A_{G} f(\xi)=\frac{1}{\pi} \int_{\mathbb{C} \backslash \bar{D}} f(z) \frac{\partial^{2}}{\partial \xi \partial z} \ln \frac{\varphi(z)-\varphi(\xi)}{z-\xi} d v(z), \quad f \in \overline{B_{2}(\mathbb{C} \backslash \bar{D})}
$$

Для краткости приведем лишь теоремы и леммы, используемые в этом случае.

Теорема В' (Грунский). Для того чтобы мероморфная в $\mathbb{C} \backslash \bar{D}$ функиия вида $\varphi(z)=z+a_{0}+a_{1} / z+a_{2} / z^{2}+\cdots$ была однолистна в $\mathbb{C} \backslash \bar{D}$ необходимо и достаточно, чтобы выполнялось неравенство

$$
\left|\sum_{m, n=1}^{\infty} a_{m n} x_{n} x_{m}\right| \leqslant \sum_{n=1}^{\infty} \frac{\left|x_{n}\right|^{2}}{n}
$$

где числа $a_{m n}$ определяются из следующего представления функиии двух комплексных переменных $\ln ((\varphi(\xi)-\varphi(z)) /(\xi-z))$ для достаточно больших по модулю чисел $\xi$ и $z$ :

$$
\ln \frac{\varphi(\xi)-\varphi(z)}{\xi-z}=\sum_{m, n=1}^{\infty} a_{m n} \xi^{-m} z^{-n}
$$

$a\left\{x_{n}\right\}_{n=0}^{\infty}-$ произвольная последовательность комплексных чисел, для которых правая часть неравенства сходится.

Если для произвольной последовательности комплексных чисел $x_{n}$ выполняется неравенство

$$
\left|\sum_{m, n=1}^{\infty} a_{m n} x_{n} x_{m}\right| \leqslant k \sum_{n=1}^{\infty} \frac{\left|x_{n}\right|^{2}}{n}, \quad k<1
$$

то говорим, что соответствующая функция $\varphi$ удовлетворяет усиленному неравенству Грунского с константой $k$. 
Лемма $4^{\prime}$. Пусть $\varphi(z)=z+a_{0}+a_{1} / z+a_{2} / z^{2}+\cdots-$ конформное отобрахсение области $\mathbb{C} \backslash \bar{D}$ на область $G$. Оператор $A_{G}$ действует из пространства $\overline{B_{2}(\mathbb{C} \backslash \bar{D})}$ в $B_{2}(\mathbb{C} \backslash \bar{D})$,

$$
A_{G} f(\xi)=\frac{1}{\pi} \int_{\mathbb{C} \backslash \bar{D}} f(z) \frac{\partial^{2}}{\partial \xi \partial z} \ln \frac{\varphi(z)-\varphi(\xi)}{z-\xi} d v(z)
$$

Следующие условия әквивалентны:

1) функиия $\varphi$ удовлетворяет усиленному условию Грунского с константой $k<1$;

2) для любой функиии $f \in \overline{B_{2}(\mathbb{C} \backslash \bar{D})}$ выполнено условие

$$
\left|\left(A_{G} f, \bar{f}\right)_{B_{2}(\mathbb{C} \backslash \bar{D})}\right| \leqslant k\|f\|_{B_{2}(\mathbb{C} \backslash \bar{D})}^{2}
$$

\section{СПИСОК ЦИТИРОВАННОЙ ЛИТЕРАТУРЫ}

[1] Державец Б. А.Пространства функций, аналитических в выпуклых областях $\mathbb{C}^{n}$ и имеющих заданное поведение вблизи границы // Докл. АН СССР. 1984. Т. 276. №6. С. 1297-1300.

[2] Любарский Ю. И. Теорема Винера-Пэли для выпуклых множеств // Изв. АН АрмССР. Матем. 1988. Т. 62. №2. С. 162-172.

[3] Луценко В. И., Юлмухаметов Р. С. Обобщение теоремы Винера-Пэли на функционалы в пространствах Смирнова // Тр. МИАН. 1991. Т. 200. С. 245-254.

[4] Напалков В. В. (мл.), Юлмухаметов Р. С. О преобразовании Коши функционалов на пространстве Бергмана // Матем. сб. 1994. Т. 185. № 7. С. 77-86.

[5] Köthe G. Dualität in der Funktionentheorie // J. Reine Angew. Math. 1953. V. 191. № 1/2. P. 30-49.

[6] Хёрмандер Л. Введение в теорию функций нескольких комплексных переменных. М.: Мир, 1986.

[7] Юлмухаметов Р.С. Пространство аналитических функций, имеющих заданный рост вблизи границы // Матем. заметки. 1982. Т. 32. №1. С. 41-57.

[8] Напалков В. В. Пространства аналитических функций заданного роста вблизи границы // Изв. АН СССР. Сер. матем. 1987. Т. 51. № 2. С. 287-305.

[9] Епифанов О.В.Двойственность одной пары пространств аналитических функций ограниченного роста // Докл. АН СССР. 1991. Т. 319. №6. С. 1297-1300.

[10] Merenkov S. A. On the Cauchy transform of the Bergman space // Математическая физика, анализ, геометрия. Вып. 4. Харьков, 1999.

[11] Calderon A.P. Cauchy integrals on Lipschitz curves and related operators // Proc. Mat. Acad. Sci. USA. 1977. P. 1324-1327.

[12] Coifman R.R., McIntosh A., Meyer Y. L'intégrale de Cauchy définit un opératuer bourné sur $L^{2}$ pour les courbes Lipschitziennes // Annals of Math. 1982. V. 116. № 2.

[13] Стейн И. Сингулярные интегралы и дифференциальные свойства функций. М.: Мир, 1973.

[14] Голузин Г. М. Геометрическая теория функций комплексного переменного. М.: Наука, 1966.

[15] Крушкаль С. Л., Кюнау Р. Квазиконформные отображения - новые методы и приложения. Новосибирск: Наука, 1984.

[16] Гайер Д. Лекции по теории аппроксимации в комплексной области. М.: Мир, 1987.

(В.В. Напалков) Институт математики с ВЦ Уфимского научного центра РАН 